\title{
A New Application of the Hill Repressor Function: Automatic Control of a Conic Tank Level and Local Stability Analysis
}

\author{
José Ricardo Pérez-Correa, ${ }^{1}$ Gastón Lefranc, ${ }^{2}$ and Mario Fernández-Fernández ${ }^{3}$ \\ ${ }^{1}$ Departamento de Ingeniería Química y Bioprocesos, Pontificia Universidad Católica de Chile, Avenida Vicuña Mackenna 4860, \\ 7820436 Santiago, Chile \\ ${ }^{2}$ Escuela de Ingeniería Eléctrica, Pontificia Universidad Católica De Valparaíso, Avenida Brasil 2950, 2340000 Valparaíso, Chile \\ ${ }^{3}$ Departamento de Tecnologías Industriales, Universidad de Talca, Camino a Los Niches km 1, 3440000 Curicó, Chile
}

Correspondence should be addressed to José Ricardo Pérez-Correa; perez@ing.puc.cl

Received 12 March 2015; Accepted 27 April 2015

Academic Editor: Sergio Preidikman

Copyright ( 2015 José Ricardo Pérez-Correa et al. This is an open access article distributed under the Creative Commons Attribution License, which permits unrestricted use, distribution, and reproduction in any medium, provided the original work is properly cited.

\begin{abstract}
The Hill function is commonly used as a building block to model different dynamic patterns found in the response of genetic regulatory circuits within microorganisms and cells. These circuits are characterized by fast response and robustness against unmeasured disturbances. Therefore, microorganisms and cells can survive even if they are subjected to strong changes in their environment. However, as far as we know, the Hill function has not been used before to design process control systems. In this work, the repressor Hill function is applied to control the level of a conic tank. To eliminate the offset, we added integrative action. A local analysis was applied to define stability limits for the control parameters. A cost function that includes the error and the control effort was used to compare the performance of the Hill control against a standard PI and a PID-Dahlin controller.
\end{abstract}

\section{Introduction}

The Hill equation has shown many interesting properties and has been applied to model several dynamic systems in biosciences, pharmacology, biology, and physicochemistry. In biosciences, this equation has been used to fit a model to experimental or clinical data when the relationship between two sets of variables was saturated and nonlinear [1-3]. In physics, chemistry, and biochemistry, this equation can describe thermodynamic equilibrium, where each parameter of the model has a physical meaning $[4,5]$. In pharmacology, the Hill equation may be used as a probabilistic expression of time-dependent biological events such as adverse reaction, toxicity, or death. In addition, this equation has been extensively used to analyze quantitative drug-receptor relationships. Many pharmacokinetic-pharmacodynamic models have used the Hill equation to describe nonlinear drug dose-response relationships [6-10].

In addition, the Hill function is commonly used as a building block to model different dynamic patterns found in the response of genetic regulatory circuits within microorganisms and cells. These circuits are characterized by fast response and robustness against disturbances. Therefore, microorganisms and cells can survive even if they are subjected to strong changes in their environment [11-14]. The Hill function can model several genetic regulatory mechanisms within cells. In particular, two basic mechanisms can be identified in transcription networks. The activation mechanism operates when the transcription factor increases the gene transcription rate (protein production rate associated with the gene). The activator Hill function represents this mechanism well: the protein level can be controlled by the signaling molecule. The repressor mechanism (that represses the binding and decreases the transcription rate) can be modelled with the repressors' Hill function. The higher the repressor concentration is, the higher the probability that a repressor molecule binds to the operator site is. Consequently, the expression level is more and more repressed with increasing repressor levels. Half-maximal repression 
occurs when the concentration of active repressors equals the repression coefficient [15].

Although the Hill equation is widely used, many of its properties are not all well known. The nonlinear Hill equation can deal properly with the control of several chaotic systems. The existence of periodic solutions in particular situations has been recently proved. Other problems that are under research related with the Hill equation are, for instance, the control of chaos by means of the transient effects minimization or by synchronization. Therefore, more interesting applications of the Hill equation can be found exploring these areas [16, 17].

As far as we know, the Hill function has not been used before to design process control systems. In this paper, the repressor Hill function is applied to control the level of a conic tank. To eliminate the offset, we added integrative action. A cost function that includes the error and the control effort was used to compare the performance of the Hill control against a standard PI and a PID-Dahlin controller [18].

\section{The Hill Controller}

The Hill function is commonly used to model several genetic regulatory mechanisms within cells. In particular, two basic mechanisms can be identified in transcription networks [1]. The activation mechanism operates when the transcription factor increases the gene transcription rate (protein production rate associated with that gene). The activator Hill function represents this mechanism well:

$$
H_{a}(y)=\frac{\beta y^{n}}{K^{n}+y^{n}}, \quad y \in R .
$$

In $(1) H_{a}(y)$ is the protein production rate and $y$ is the concentration of the transcription factor; $K, \beta$, and $n$ are model parameters. $K$, the activation coefficient, is the threshold value of $y$ that activates the mechanism; $\beta$, the maximal expression level, is the maximum response value; and $n$, the Hill coefficient, defines the response steepness.

In turn, the repressor mechanism operates when the transcription factor reduces the gene transcription rate. This mechanism can be modelled by the repressor Hill function:

$$
H_{r}(y)=\frac{\beta}{1+(y / K)^{n}}, \quad y \in R .
$$

Model parameters are similar to those in (1); however, (2) is a decreasing function; therefore the maximum expression is obtained for $y=0$.

Equation (2) can be adapted for process control to profit from the fast response and robustness that characterize genetic regulatory circuits. Therefore, the following Hill controller can be proposed:

$$
\begin{aligned}
u(t) & =\frac{\beta}{1+\left[y(t) / y^{*}\right]^{n}}=\frac{\beta}{1+\left[\left(y^{*}-e(t)\right) / y^{*}\right]^{n}} \\
& =\frac{\beta y^{* n}}{y^{* n}+\left[y^{*}-e(t)\right]^{n}},
\end{aligned}
$$

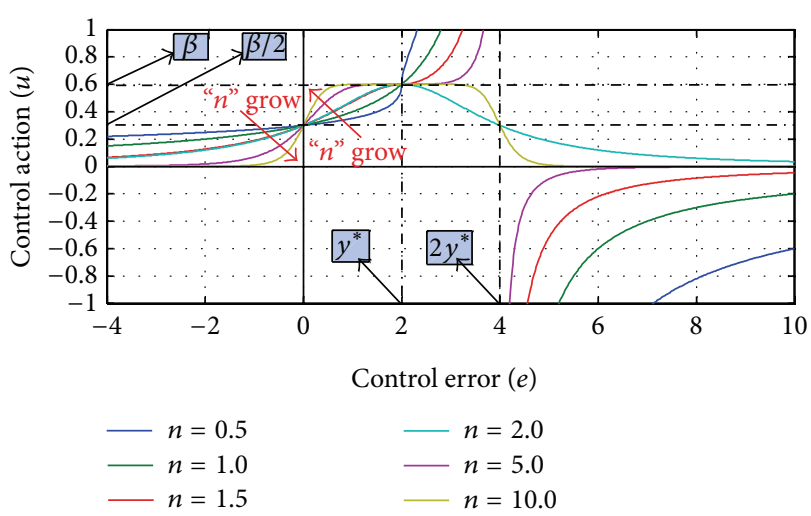

FIGURE 1: Hill control versus control error for different values of $n$. Fixed parameters: $\beta=0.6, y^{*}=2$.

where $y$ is the controlled variable, $y^{*}$ is the set point, $e(t)$ is the control error, $\beta$ is twice the initial steady state control effort (considering that the process is initially at the desired operating point), and $n$ is the steepness index. Next, we analyze the impact of the model parameters on the behavior of this control algorithm.

In Figure 1 we see that $u=\beta$ when $e=y^{*}$ where the control action presents an extreme point (maximum or inflection point). In addition, the control action is $u=\beta / 2$ when $e=0$ and $e=2 y^{*}$ (if $n$ is even). However, if $n$ is odd or not an integer, the control action diverges $(u \rightarrow \infty)$ when $e=2 y^{*}$. For stable control systems, it is expected that the control error would be less than the set point $\left(e<y^{*}\right)$. Within this error range, for $n>1$, the control action will be sigmoidal with an inflection point at $(e=0 ; u=\beta / 2)$. Finally, if $|e| \gg\left|y^{*}\right|$ the control action approaches 0 for all values of $n$.

The controller proposed in (3) will not present an offset if the value of $\beta$ (twice the control action that keeps $y$ in its set point $y^{*}$ ) is known. However, since $\beta$ is generally unknown for every possible set point value, an offset will be generated and therefore an integral control action will be needed to eliminate it.

Therefore, an integral Hill controller is proposed:

$$
u(t)=\frac{\beta y^{* n}}{y^{* n}+\left[y^{*}-e(t)\right]^{n}}+I \int_{0}^{t} e(t) d t .
$$

As analyzed below, the stability of this controller will depend on the values of $\beta$ and $I$.

\section{PID Controllers}

If $G_{P}(s)$ is the plant transfer function, $G_{S}(s)$ is the sensor transfer function, $G_{V}(s)$ is the valve transfer function, and $G_{C}(s)$ is the controller transfer function, the closed-loop transfer function, $G_{C L}(s)$, is given by

$$
G_{\mathrm{CL}}(s)=\frac{G_{C} G_{V} G_{P} G_{S}}{1+G_{C} G_{V} G_{P} G_{S}}=\frac{Y_{S}(s)}{Y^{*}(s)} .
$$


The controller $G_{C}(s)$ that achieves the desired set point trajectory, $Y^{*}(s)$, is

$$
G_{C}(s)=\frac{Y_{S}(s) / Y^{*}(s)}{G_{V} G_{P} G_{S}\left[1-Y_{S}(s) / Y^{*}(s)\right]} .
$$

In the Dahlin controller [2], $G_{\mathrm{CL}}(s)$ is a first-order plus dead time (FOPDT) transfer function, where the dead time is given by the process transfer function,

$$
\frac{Y_{S}(s)}{Y^{*}(s)}=\frac{e^{-\theta_{P} s}}{\tau_{C} s+1},
$$

and $\tau_{C}$ is a tuning parameter. Replacing (7) in (6) yields

$$
G_{C}(s)=\frac{e^{-\theta_{P} s}}{G_{V} G_{P} G_{S}\left(\tau_{C} s+1-e^{-\theta_{P} s}\right)} .
$$

If the open-loop transfer function can be approximated as a FOPDT,

$$
G_{V} G_{P} G_{S}(s) \approx \frac{K_{P}}{\tau_{P} s+1} e^{-\theta_{P} s},
$$

replacing (9) in (8) results in

$$
G_{C}(s)=\frac{\tau_{P} s+1}{K_{P}\left(\tau_{C} s+1-e^{-\theta_{P} s}\right)} .
$$

Using a first-order Padé approximation for dead time finally yields

$$
G_{C}(s)=\frac{\tau_{P}}{K_{P}\left(\tau_{C}+\theta_{P}\right)}\left(1+\frac{1}{\tau_{P} s}\right)\left(\frac{\theta_{P} s / 2-1}{\alpha \theta_{P} s / 2+1}\right) .
$$

This corresponds to a commercial proportional-integralderivative (PID) algorithm, with a filter in the derivative action. The controller parameters are defined by the process transfer function and the only tuning parameter (the closedloop time constant),

$$
\begin{aligned}
K_{C} & =\frac{\tau_{P}}{K_{P}\left(\tau_{C}+\theta_{P}\right)} ; \\
\tau_{I} & =\tau_{P} ; \\
\tau_{D} & =\frac{\theta_{P}}{2} ; \\
\alpha & =\frac{\tau_{C}}{\left(\tau_{C}+\theta_{P}\right)} .
\end{aligned}
$$

A standard PID tuning method, still applied in industry, is the Open-Loop Ziegler \& Nichols rule [2]:

$$
\text { For PI control: } \begin{aligned}
K_{C} & =\frac{0.9 \tau_{P}}{K_{P} \theta_{P}} ; \\
\text { For PID control: } \tau_{I} & =3.33 \theta_{P}, \\
K_{C} & \frac{1.2 \tau_{P}}{K_{P} \theta_{P}} ; \\
\tau_{I} & =2 \theta_{P} ; \\
\tau_{I} & =0.5 \theta_{P} .
\end{aligned}
$$

\section{Local Stability Analysis of the Integral Hill Controller}

To perform a local stability analysis, we have to linearize the Hill controller (4):

$$
u(t)=\frac{n \beta\left[\left(y^{*}-e\left(t_{0}\right)\right) / y^{*}\right]^{n-1}}{\left\{1+\left[\left(y^{*}-e\left(t_{0}\right)\right) / y^{*}\right]^{n}\right\}^{2}} e(t)+I \int_{0}^{t} e(t) d t .
$$

If we linearize (4) around the set point, $e\left(t_{0}\right)=e_{0}=0$,

$$
u(t)=\frac{n \beta}{4 y^{*}} e(t)+I \int_{0}^{t} e(t) d t .
$$

Equation (15) is a proportional-integral (PI) control, where the proportional gain depends inversely on the set point value and directly on the Hill coefficient and the maximal expression level.

Applying Laplace transform, the following controller transfer function is obtained:

$$
G_{c}(s)=\frac{n \beta}{4 y^{*}}+\frac{I}{s} .
$$

If we consider that the plant model (including sensor and valve dynamics) can be approximated by a first-order plus dead time transfer function, the characteristic equation is

$$
1+G_{P} G_{c}(s)=1+\left(\frac{K_{P} e^{-\theta_{P} s}}{\tau_{P} s+1}\right)\left(\frac{n \beta}{4 y^{*}}+\frac{I}{s}\right)=0 .
$$

In addition, the dead time can be approximated by firstorder Padé,

$$
\begin{aligned}
& \left(\tau_{P} s+1\right)\left(1+\frac{\theta_{P}}{2} s\right)+K_{P}\left(1-\frac{\theta_{P}}{2} s\right)\left(\frac{n \beta}{4 y^{*}}+\frac{I}{s}\right) \\
& \quad=0 .
\end{aligned}
$$

Reordering this equation yields

$$
\begin{aligned}
\frac{\tau_{P} \theta_{P}}{2} s^{3} & +\left(\tau_{P}+\frac{\theta_{P}}{2}-\frac{n \beta \theta_{P} K_{P}}{8 y^{*}}\right) s^{2} \\
& +\left(1-\frac{\theta_{P}}{2} K_{P} I+\frac{n \beta K_{P}}{4 y^{*}}\right) s+K_{P} I=0 .
\end{aligned}
$$

The Routh stability criterion can be more easily applied using the following definitions:

$$
\begin{aligned}
& a_{0}=\frac{\tau_{P} \theta_{P}}{2} ; \\
& a_{1}=\tau_{P}+\frac{\theta_{P}}{2}\left(1-\frac{n \beta K_{P}}{4 y^{*}}\right) ; \\
& a_{2}=\left(1-\frac{\theta_{P}}{2} K_{P} I+\frac{n \beta K_{P}}{4 y^{*}}\right) ; \\
& a_{3}=K_{P} I,
\end{aligned}
$$


resulting in the following stability conditions:

$$
\begin{aligned}
a_{0}= & \frac{\tau_{P} \theta_{P}}{2}>0 \\
a_{1}= & \tau_{P}+\frac{\theta_{P}}{2}\left(1-\frac{n \beta K_{P}}{4 y^{*}}\right)>0, \\
a_{4}= & \frac{a_{1} a_{2}-a_{0} a_{3}}{a_{1}}>0 \longrightarrow a_{1} a_{2}-a_{0} a_{3}>0, \\
a_{1} a_{2}- & a_{0} a_{3} \\
= & {\left[\tau_{P}+\frac{\theta_{P}}{2}\left(1-\frac{n \beta K_{P}}{4 y^{*}}\right)\right]\left(1-\frac{\theta_{P}}{2} K_{P} I+\frac{n \beta K_{P}}{4 y^{*}}\right) } \\
& -\left(\frac{\tau_{P} \theta_{P}}{2}\right)\left(K_{P} I\right)>0, \\
\therefore & \tau_{P}+\frac{\theta_{P}}{2}+\frac{n \beta K_{P} \tau_{P}}{4 y^{*}}+\frac{n \beta I K_{P}{ }^{2} \theta_{P}^{2}}{16 y^{*}} \\
& -\left(I K_{P} \theta_{P} \tau_{P}+\frac{\theta_{P}{ }^{2}}{4} K_{P} I+\frac{n^{2} \beta^{2} K_{P}{ }^{2} \theta_{P}}{32 y^{*}}\right)>0 .
\end{aligned}
$$

Then $a_{5}=a_{3}=K_{P} I>0$.

These conditions can be summarized as

$$
\begin{aligned}
\beta & <\frac{8 y^{*}}{n \theta_{P} K_{P}}\left(\tau_{P}+\frac{\theta_{P}}{2}\right), \\
I>0 & \\
\tau_{P} & +\frac{\theta_{P}}{2}+\frac{n \beta K_{P} \tau_{P}}{4 y^{*}}+\frac{n \beta I K_{P}{ }^{2} \theta_{P}^{2}}{16 y^{*}} \\
& -\left(I K_{P} \theta_{P} \tau_{P}+\frac{\theta_{P}{ }^{2}}{4} K_{P} I+\frac{n^{2} \beta^{2} K_{P}{ }^{2} \theta_{P}}{32 y^{*}}\right)>0 .
\end{aligned}
$$

\section{The Process Model}

To assess the applicability of the proposed Hill controller, a conic tank model was used:

$$
\begin{aligned}
\frac{d h}{d t} & =\frac{1}{\pi}\left(\frac{H}{R}\right)^{2}\left(\frac{1}{h^{2}} F e-\varphi h^{-3 / 2}\right) \\
& =\gamma\left(\frac{1}{h^{2}} F e-\varphi h^{-3 / 2}\right),
\end{aligned}
$$

where $F e$ is the inlet flow rate, $h$ is the tank level, $\varphi$ is the discharge constant of the tank, and $H$ and $R$ are the height and the maximum radius of the tank.

The sensor is modelled as a FOPDT transfer function and the inlet valve is linear, with installed characteristic given by

$$
F(u)=\frac{F_{\max } u}{15850 \sqrt{\alpha+(1-\alpha) u^{2}}}
$$

TABLE 1: Model parameters.

\begin{tabular}{lccc}
\hline Parameter [3] & Description & Value & Units \\
\hline$\gamma$ & Tank parameter & 0.262 & - \\
$\varphi$ & Tank parameter & 0.5 & $\mathrm{~m}^{2.5} \cdot \mathrm{s}^{-1}$ \\
$K_{s}$ & Sensor gain & 1 & - \\
$\tau_{s}$ & Sensor time constant & 1 & $\mathrm{~S}$ \\
$\theta_{s}$ & Sensor time delay & 3 & $\mathrm{~S}$ \\
$C_{v}$ & Valve coefficient & 1200 & $\mathrm{gpm} \cdot \mathrm{psi}^{0.5}$ \\
$K_{1}$ & Pressure drop constant & 800 & $\mathrm{gpm} \cdot \mathrm{psi}^{0.5}$ \\
$P_{0}$ & Downstream pressure & 27 & $\mathrm{psi}$ \\
$P_{1}$ & Upstream pressure & 1000 & $\mathrm{psi}$ \\
\hline
\end{tabular}

where

$$
\begin{gathered}
F_{\max }=K_{1} \sqrt{P_{1}-P_{1_{\text {min }}}} \\
\alpha=\frac{P_{1_{\min }}-P_{0}}{P_{1}-P_{0}} ; \\
P_{1_{\text {min }}}=\frac{K_{1}^{2} P_{1}+C_{v}^{2} P_{0}}{K_{1}^{2}+C_{v}^{2}} .
\end{gathered}
$$

\section{Simulation Results}

This model was simulated in Simulink with the model parameters shown in Table 1.

The performance of the integral Hill controller is compared with a PI controller tuned with Z\&N (a Z\&N tuned PID was unstable), with a PID-Dahlin and with a PI tuned with the Matlab Control Toolbox. Figure 2 shows the simulation structure in Simulink.

Using the TRIM and LINMOD Matlab functions, we linearized the system around the steady state $h_{0}=1 \mathrm{~m}$. The controller output in this condition takes the value of $u=$ 0.2233 , and the approximate transfer function is

$$
\left.G_{P}(s)\right|_{1.0 \mathrm{~m}}=\frac{8.05}{4.53 s+1} e^{-3.0 s}
$$

To compare the performance of the standard controllers with that of the integrative Hill controller proposed in this work, we applied a series of set point changes between $0.7 \mathrm{~m}$ and $1.3 \mathrm{~m}$ and evaluated the following performance index $J$ that takes into account the error and the control effort:

$$
J=\int_{0}^{\infty}|e(t)| d t+10 \int_{0}^{\infty}\left|\frac{d u(t)}{d t}\right| d t .
$$

The range of possible Hill controller parameters can be obtained from the stability conditions above (22). Considering $y^{*}=h_{0}=1 \mathrm{~m}, n=1$ (larger values make tuning more difficult), and replacing nominal plant parameters,

$$
\begin{aligned}
& I>0 ; \\
& \beta<15.48,
\end{aligned}
$$

$$
9.1112 \beta-11.1147 I-0.6082 \beta^{2}+0.3649 \beta I+4.6749
$$


TABLE 2: Controller parameters.

\begin{tabular}{|c|c|c|c|c|}
\hline Controller & Parameter & Description & Value & $J$ \\
\hline \multirow{2}{*}{ PI Z\&N (13) } & $K_{C}$ & Proportional gain & 1.6854 & \multirow{2}{*}{$2.510^{4}$} \\
\hline & $K_{I}$ & Integral gain & 1.6854 & \\
\hline \multirow{2}{*}{ PI (Matlab tuning) } & $K_{C}$ & Proportional gain & 0.051786 & \multirow{2}{*}{29.7} \\
\hline & $K_{I}$ & Integral gain & 0.014306 & \\
\hline \multirow{4}{*}{ PID-Dahlin (14) } & $K_{C}$ & Proportional gain & 0.1478 & \multirow{4}{*}{24.4} \\
\hline & $\tau_{I}$ & Integral time & 4.5249 & \\
\hline & $\tau_{D}$ & Derivative time & 0.15 & \\
\hline & $\tau_{C}$ & Closed-loop time constant & 0.35 & \\
\hline \multirow{3}{*}{ Hill controller } & $\beta$ & Upper value of control action & 0.4466 & \multirow{3}{*}{27.7} \\
\hline & $K_{1}$ & Integral gain & 0.017 & \\
\hline & $n$ & Hill index & 1 & \\
\hline
\end{tabular}

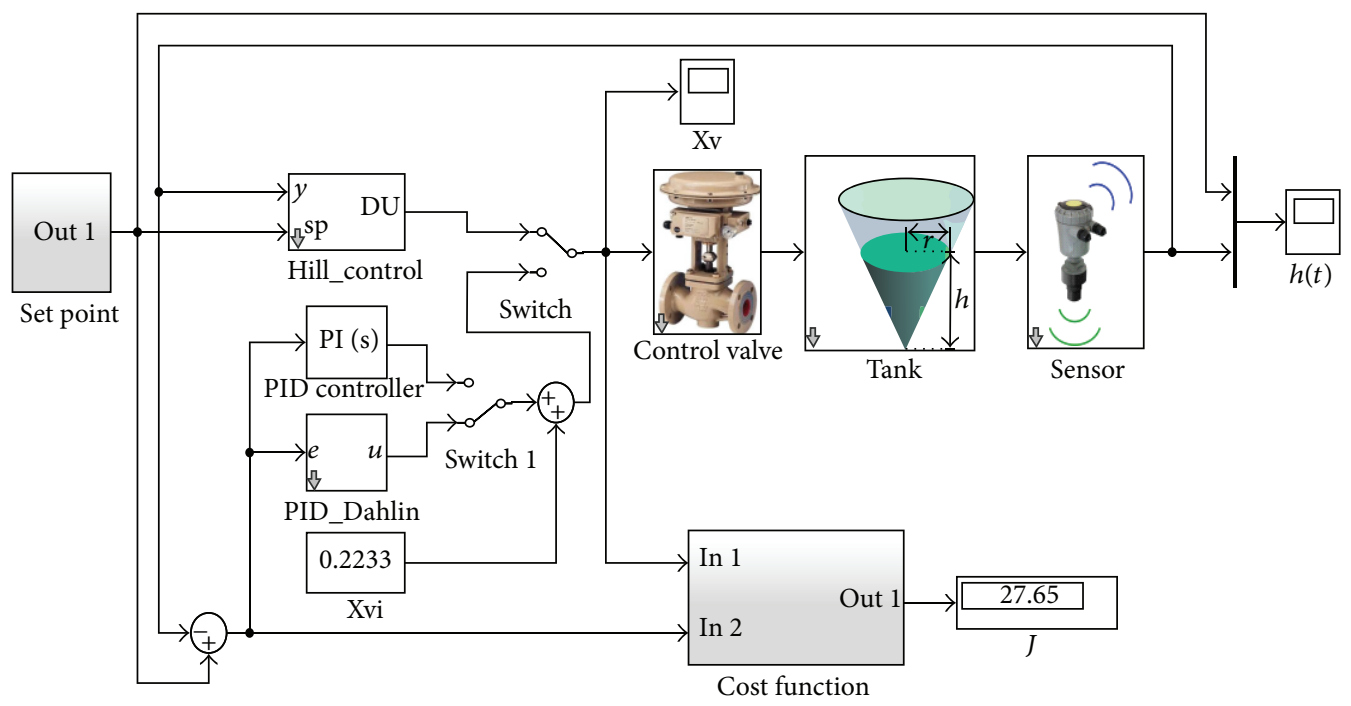

FiguRE 2: Tank level control simulation in Simulink.

The value of $\beta$ can be obtained from the initial control action; that is, $\beta=2 \cdot 0.2233=0.4466$. The value of $I$ can be obtained by trial and error, taking values between $I=0$ and $I=0.79$ (obtained from 27 for $\beta=0.4466$ ). After a few iterations, good control performance is achieved with $I=0.017$. The value of $\tau_{C}$ in the PID-Dahlin controller was also tuned by trial and error. The PI controller was tuned with Ziegler-Nichols and with the automatic tuning method of the Matlab Control Toolbox. Table 2 summarizes the parameter values applied in the simulations and their corresponding performance index values.

Finally, Figure 3 shows the results of the Hill controller.

It can be seen that the Hill controller presents good performance with minimum process information and tuning only one parameter $(I)$ with moderate effort.

\section{Conclusions}

A new control algorithm based on the Hill repressor function that uses only one tuning parameter has been presented. The function is applied to control the level of a conic tank. To eliminate the offset, an integrative action has been added. A cost function, which includes the error and the control effort, was used to compare the performance of the Hill control against a standard PI and a PID-Dahlin controller.

The algorithm was tested using square wave set point changes. Moreover, a local stability analysis was performed and it found the bounds of the Hill controller tuning parameters to ensure a stable closed loop.

The Hill controller is easy to tune by trial and error and can perform almost as well as a PID-Dahlin controller and better than an optimally tuned PI controller. It can be seen that the Hill controller presents good performance with minimum process information and by tuning only one parameter $(I)$ with moderate effort. The Hill controller presents similar performance compared to other controllers that required much more process information and that are more difficult to apply by nonexpert operators.

Future work will include testing the integrative Hill controller in more challenging control systems, to derive and assess a controller based on the Hill activator function and apply Lyapunov theory to derive more general and precise stability conditions. 


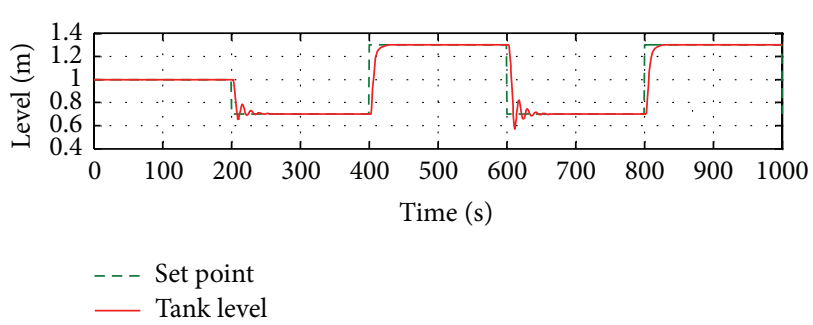

(a)

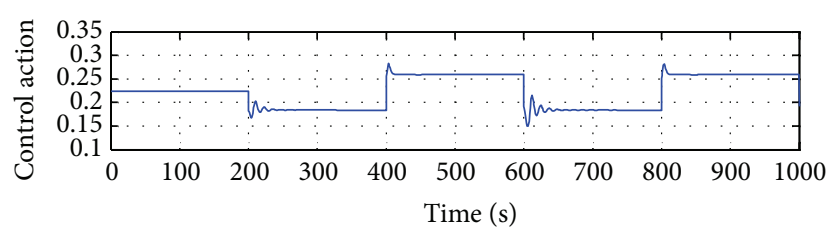

(b)

FIGURE 3: Tank level integrative Hill control. Process variable (a) and manipulated variable (b).

\section{Conflict of Interests}

The authors declare that there is no conflict of interests regarding the publication of this paper.

\section{References}

[1] S. Goutelle, M. Maurin, F. Rougier et al., "The Hill equation: a review of its capabilities in pharmacological modelling," Fundamental \& Clinical Pharmacology, vol. 22, no. 6, pp. 633648, 2008.

[2] D. Chu, N. R. Zabet, and B. Mitavskiy, "Models of transcription factor binding: sensitivity of activation functions to model assumptions," Journal of Theoretical Biology, vol. 257, no. 3, pp. 419-429, 2009.

[3] U. Alon, An Introduction to Systems Biology-Design Principles of Biological Networks, Chapman \& Hall, CRC Press, Boca Raton, Fla, USA, 2007.

[4] G. F. Froment, "On fundamental kinetic equations for chemical reactions and processes," Current Opinion in Chemical Engineering, vol. 5, pp. 1-6, 2014.

[5] R. Cammack, T. Atwood, P. Campbell et al., Oxford Dictionary of Biochemistry and Molecular Biology, Oxford University Press, 2nd edition, 2008.

[6] W. Krzyzanski and W. J. Jusko, "Mathematical formalism for the properties of four basic models of indirect pharmacodynamic responses," Journal of Pharmacokinetics and Biopharmaceutics, vol. 25, no. 1, pp. 107-123, 1997.

[7] L. Barbanti and B. C. Damasceno, "Control aspects in nonlinear Hill's equation," Communications in Nonlinear Science and Numerical Simulation, vol. 16, no. 5, pp. 2328-2331, 2011.

[8] S. Goutelle, M. Maurin, F. Rougier et al., "The Hill equation: a review of its capabilities in pharmacological modelling," Fundamental and Clinical Pharmacology, vol. 22, no. 6, pp. 633648, 2008.

[9] R. Gesztelyi, J. Zsuga, A. Kemeny-Beke, B. Varga, B. Juhasz, and A. Tosaki, "The Hill equation and the origin of quantitative pharmacology," Archive for History of Exact Sciences, vol. 66, no. 4, pp. 427-438, 2012.

[10] D. Colquhoun, "The quantitative analysis of drug-receptor interactions: a short history," Trends in Pharmacological Sciences, vol. 27, no. 3, pp. 149-157, 2006.

[11] D. A. Oyarzún, J. Lugagne, and G. V. Stan, "Noise propagation in synthetic gene circuits for metabolic control," ACS Synthetic Biology, vol. 4, no. 2, pp. 116-125, 2015.

[12] D. A. Oyarzún and G.-B. V. Stan, "Synthetic gene circuits for metabolic control: design trade-offs and constraints," Journal of the Royal Society Interface, vol. 10, no. 78, Article ID 20120671, 2013.

[13] D. Del Vecchio, "A control theoretic framework for modular analysis and design of biomolecular networks," Annual Reviews in Control, vol. 37, no. 2, pp. 333-345, 2013.

[14] R. Maas and S. Leyendecker, "Biomechanical optimal control of human arm motion," Proceedings of the Institution of Mechanical Engineers, Part K: Journal of Multi-body Dynamics, vol. 227, no. 4, pp. 375-389, 2013.

[15] T. Gedeon, K. Mischaikow, K. Patterson, and E. Traldi, "When activators repress and repressors activate: a qualitative analysis of the Shea-Ackers model," Bulletin of Mathematical Biology, vol. 70, no. 6, pp. 1660-1683, 2008.

[16] L. Barbanti and B. C. Damasceno, "Control aspects in nonlinear Hill's equation," Communications in Nonlinear Science and Numerical Simulation, vol. 16, no. 5, pp. 2328-2331, 2011.

[17] H. Coskun, "Some inverse results for Hill's equation," Journal of Mathematical Analysis and Applications, vol. 276, no. 2, pp. 833-844, 2002.

[18] B. A. Ogunaike and W. A. Ray, Process Dynamics, Modeling and Control, Oxford University Press, New York, NY, USA, 1994. 


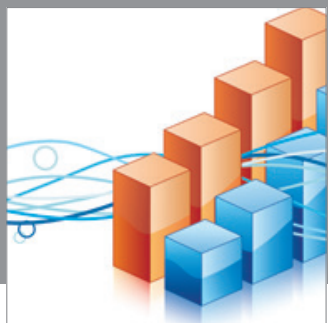

Advances in

Operations Research

mansans

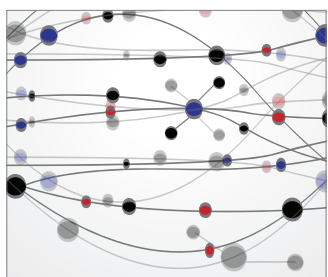

The Scientific World Journal
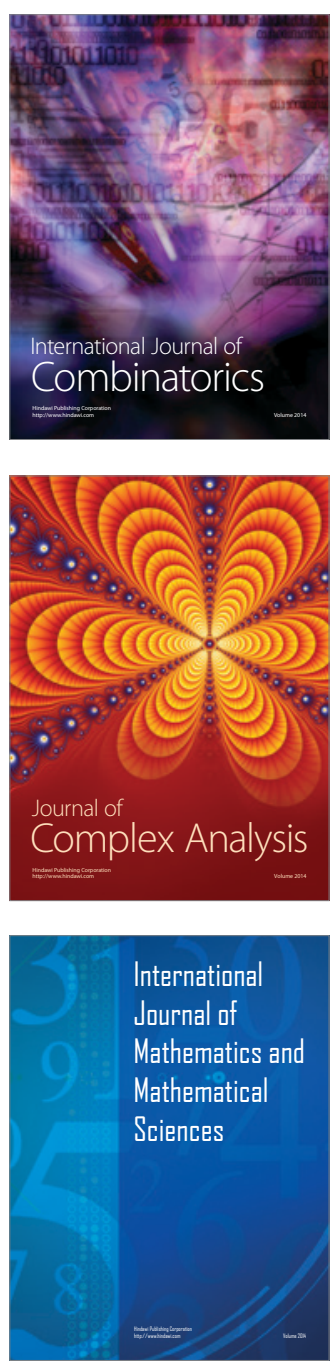
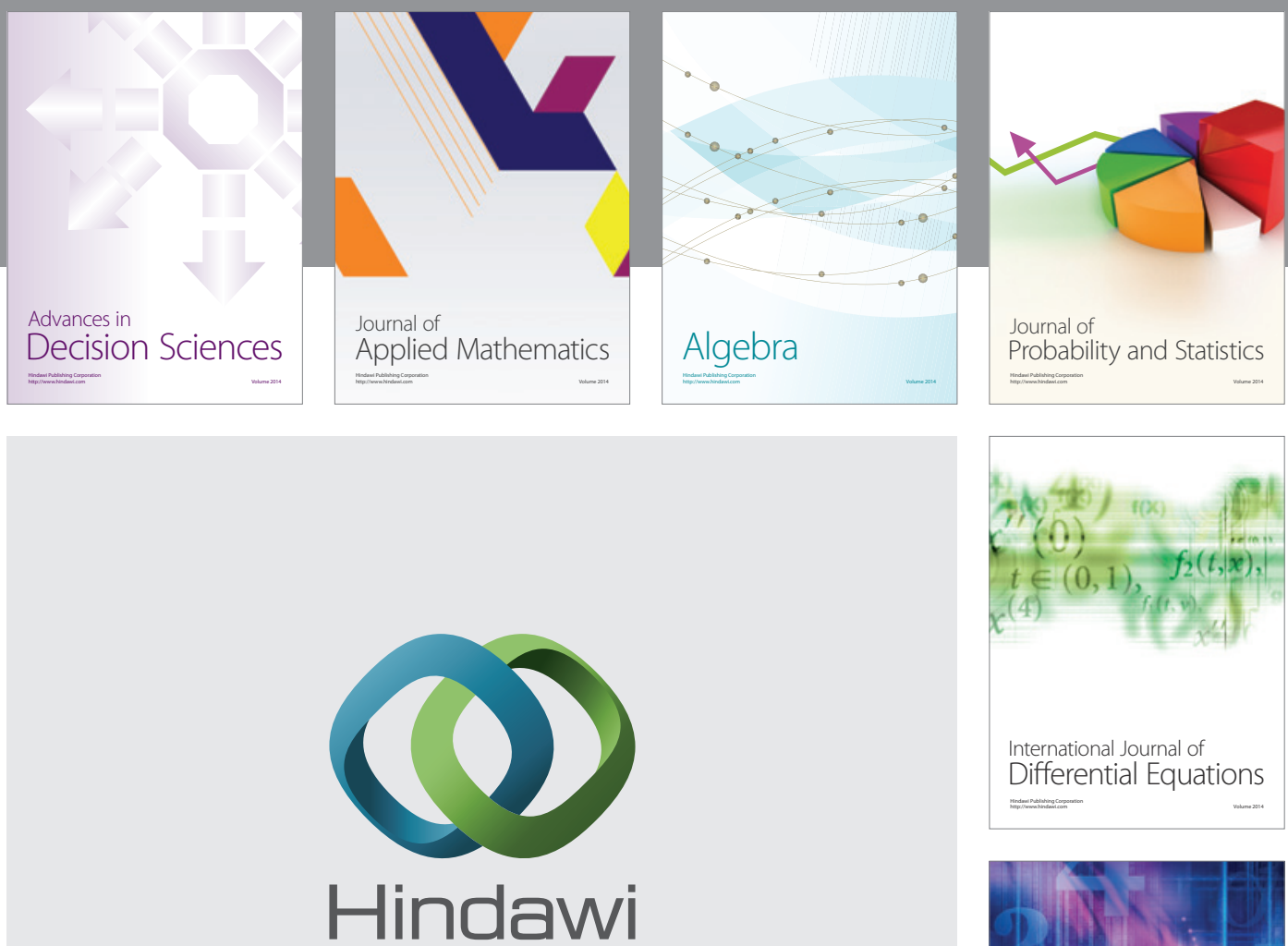

Submit your manuscripts at http://www.hindawi.com
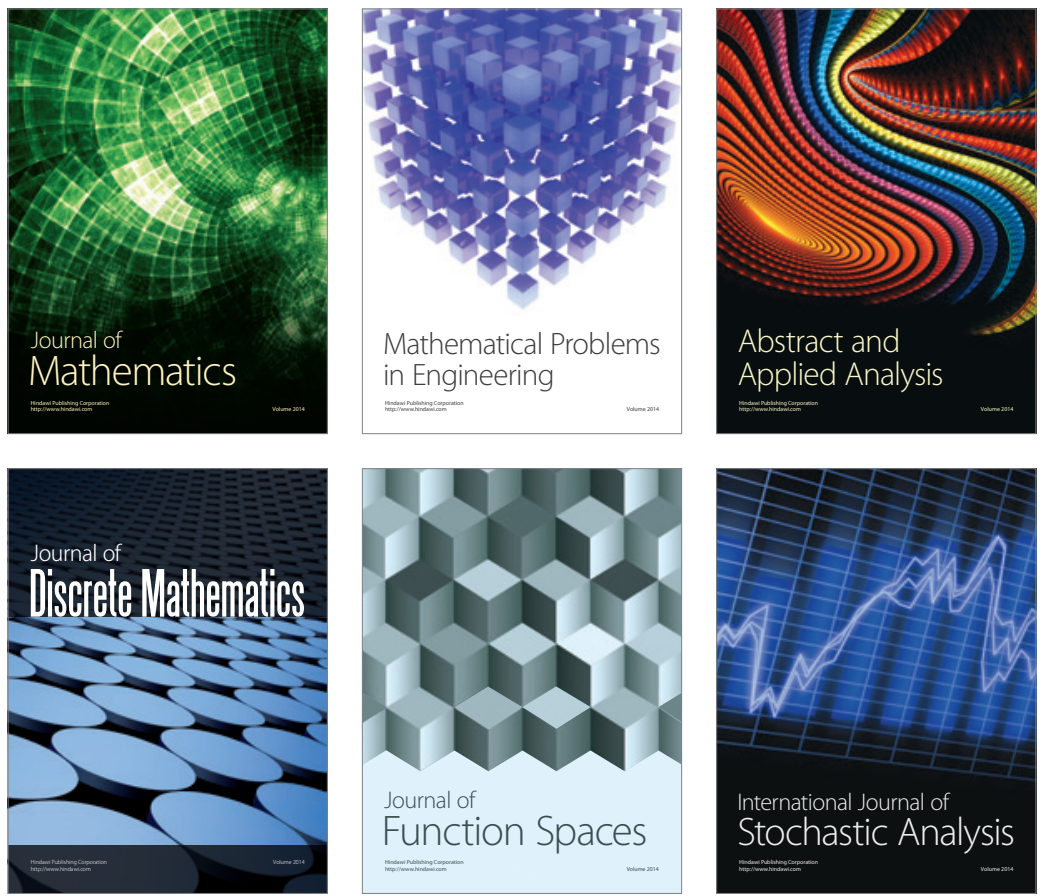

Journal of

Function Spaces

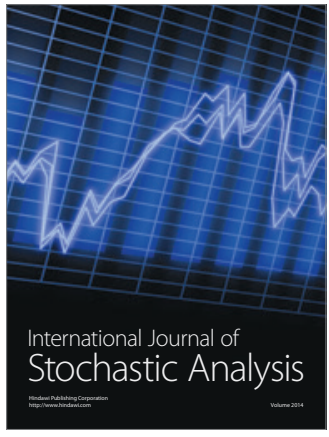

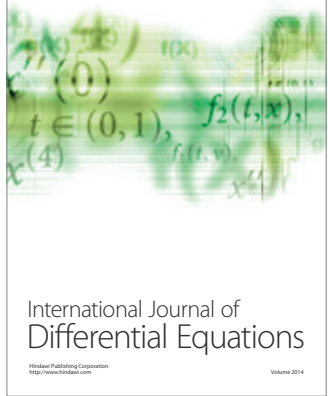
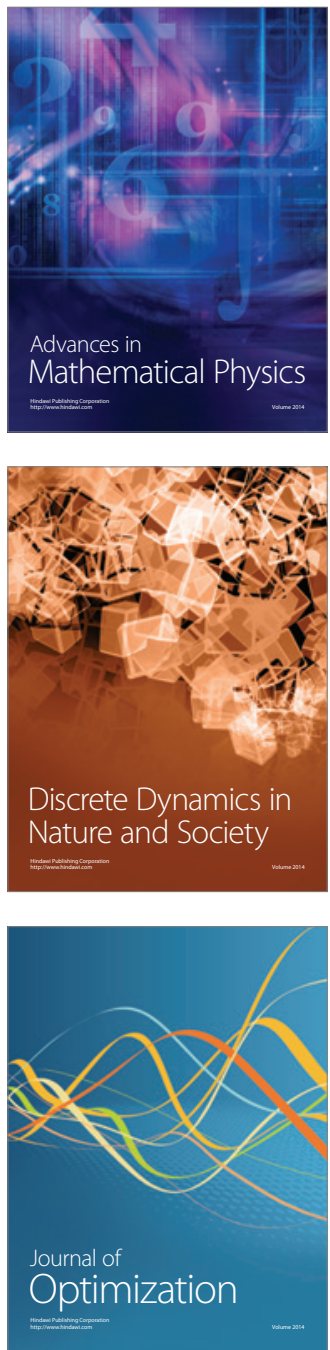